University of Massachusetts Amherst

ScholarWorks@UMass Amherst

Chemistry Department Faculty Publication Series

Chemistry

1992

\title{
Simple model of flow-injection sample transport for prediction of peak heights
}

\author{
S. R. Bysouth \\ University of Massachusetts Amherst \\ Julian Tyson \\ University of Massachusetts Amherst
}

Follow this and additional works at: https://scholarworks.umass.edu/chem_faculty_pubs

Part of the Analytical Chemistry Commons

\section{Recommended Citation}

Bysouth, S. R. and Tyson, Julian, "Simple model of flow-injection sample transport for prediction of peak heights" (1992). Analytica Chimica Acta. 1438.

Retrieved from https://scholarworks.umass.edu/chem_faculty_pubs/1438 


\title{
Simple model of flow-injection sample transport for prediction of peak heights",
}

\author{
S.R. Bysouth and J.F. Tyson * \\ Department of Chemistry, University of Massachusetts, Amherst, MA 01003 (USA)
}

(Received 23rd August 1991; revised manuscript received 9th November 1991)

\begin{abstract}
A model of the flow characteristics of a simple single-line flow-injection manifold is described and evaluated for the effects of various operating conditions on peak parameters, in particular peak height. The model is based on the mechanism for dispersion of diffusion across concentration gradients generated by laminar flow in a closed circular pipe. The flow conduit is considered to be divided into a number of segments both radially and longitudinally. The number of the segments in both dimensions is calculated from an empirically derived relationship from the initial input parameters of length, internal diameter, sample volume, detector volume and flow-rate. An iterative procedure assigns whole number values to the number of segments. Both normal and reverse procedures can be modelled. Diffusion is modelled by averaging the concentration of any one segment over those with which it has a common boundary and flow by transport of a proportion of the contents of a segment according to a parabolic velocity profile. The performance of the model was evaluated from the predicted peak heights for manifolds in which injector volume, flow-rate and tube length were varied. The predicted effect of changing the volume of the detector was examined. The model indicates that detector volume has little effect on peak shape unless the injected volume is less than the detector volume.
\end{abstract}

Keywords: Flow system; Peak height prediction; Sample transport

A number of approaches have been used to model or characterise the dispersion of an injected sample zone in a flow-injection manifold as it is transported and detected [1-17]. The approaches used so far are based on the numerical solving of Taylor's convection-diffusion equation [1], flow models involving tanks (e.g., the single, well-stirred tank [2,3], tanks in series [4,5] and tanks in parallel [6] models), empirical derivation of equations relating manifold components to their effect on peak parameters [7], a random walk diffusion model [8], a plug flow-axial dispersion model [9-12] and impulse response deconvolution techniques [13].

The effect of various manifold components at different flow-rates has been studied in great detail [14-16] and some attempts to fit model data to experimental data has been undertaken $[6,14]$. For models involving tanks, the tank mixing process is considered to be preceded by a sample transport process in which no dispersion takes place and detection occurs in an infinitely thin slice across the tank exit. This means that for these models all the manifold dispersion processes, regardless of their origin are modelled by changes in the volume(s) of the tank or tanks and the number of tanks." These models are insensitive to the effects of flow-rate on parameters such as time of first appearance because as soon as material enters the system of tanks it is considered to be instantly mixed throughout. It is also difficult to relate the model parameters to particular manifold components, such as tube length. However, this model does have utility as a basis 
of comparison of manifold designs [18] and in situations where there is considerable real mixing in tank volumes in the manifold (such as is encountered with flame atomic absorption spectrometers) [19].

Bysouth [17] suggested that a manifold could be modelled by considering all of it to be divided longitudinally into a large number of segments and modelling the transfer of material between the segments. This approach allows the dispersion processes to be considered to occur in all parts of the manifold. By considering the flow profile to be parabolic, it was shown that the trends observed with real systems, when various manifold characteristics were changed, could be reproduced by the model. No attempt was made to force the model to predict parameters such as peak arrival time, shape or height.

Golay and Attwood [20,21] considered the tube to be divided both radially and longitudinally (into a large number of segments) and modelled Poiseuille convection and radial diffusion as two repeating processes. Injection and detection was considered to be performed over narrow slices across the tube.

In this paper, these approaches of considering a flow-injection manifold as a segmented system have been extended so that the number of divisions longitudinally and radially varies with the residence time of the sample slug. This enables peak heights in real manifolds to be predicted reliably, for a number of changing conditions. The model considers diffusion and longitudinal transport by laminar flow.

\section{EXPERIMENTAL}

\section{Apparatus}

Flow-injection manifolds were constructed from $0.5 \mathrm{~mm}$ i.d. tubing (Omnifit, UK) and a rotary injection valve (Bifok, Sweden). The detector consisted of a $20-\mu \mathrm{l}, 10-\mathrm{mm}$ path-length flow cell (Philips, UK) mounted in a visible Novaspec II spectrophotometer (LKB-Pharmacia, Sweden). The carrier was water obtained from a deionisation unit (Barnstead, USA) and was pumped using a Model 180 gear pump (Micropump, USA).
A $0.25 \mathrm{~g} \mathrm{l}^{-1}$ of tartrazine (Eastman Kodak, USA) was used as a tracer and its absorbance was monitored at $426 \mathrm{~nm}$. Peaks were recorded using a SE 120 chart recorder (Asea Brown Boveri, USA) and scanned into a Macintosh SE microcomputer (Apple, USA) using a "GrayScale" scanner (MSF-3000QS, Microtech, USA). The resultant graphics file was converted into data values using the Datascan software package (Brainpower, USA).

The model programs were written in BASIC on a Cyber 870A mainframe computer (Control Data Co.) running under the VE operating system. Programs were compiled from BASIC into machine code. The Macintosh microcomputer was used as a terminal which allowed Microphone (Software Ventures, USA), the terminal program and Cricketgraph (Cricket Software, USA), a graphing program, to be run simultaneously under Multifinder (Apple, USA). This enabled the data generated by the model or using real manifolds to be captured, manipulated and plotted.

\section{Procedures}

The manifolds that were constructed are summarized in Fig. 1 and Table 1. It should be noted that the internal flow path within the valve which does not contain sample, and the part of the flow cell that is not in the light path contribute to the volume of the manifold. The dimensions of these tubes were estimated and a length of $0.5 \mathrm{~mm}$ i.d. manifold equivalent in volume was included in the measurements of tube length in Table 1. Lengths of tubing between the valve and the detector were measured, whereas the injection volumes were calculated from the mass of water contained within the loop. This was measured by filling the loop ten times, each time flushing the contents into a weighing dish using air. Flow-rates

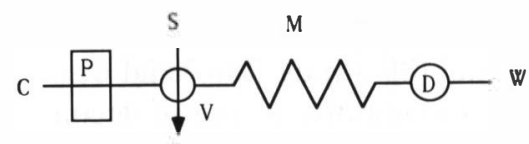

Fig. 1. Manifold diagram for the single line manifolds modeled. $\mathrm{C}$ is the carrier, $\mathrm{D}$ the detector, $\mathrm{M}$ the manifold tubing (non-coiled), $\mathrm{P}$ the gear pump, $\mathrm{S}$ the sample, $\mathrm{V}$ the injection valve, and $\mathrm{W}$ the waste. 
TABLE 1

Manifolds modelled ( $V_{\mathrm{s}}=$ sample volume; $V_{\mathrm{d}}=$ detector volume; $L_{\mathrm{m}}=$ manifold time; $F=$ flow-rate)

\begin{tabular}{lccll}
\hline $\begin{array}{l}\text { Code, } \\
\text { Fig. 4 }\end{array}$ & $\begin{array}{l}V_{\mathrm{s}} \\
(\mu \mathrm{l})\end{array}$ & $\begin{array}{l}L_{\mathrm{m}} \\
(\mathrm{mm})\end{array}$ & $\begin{array}{l}F \\
\left(\mathrm{ml} \mathrm{min}^{-1}\right)\end{array}$ & $\begin{array}{l}V_{\mathrm{d}} \\
(\mu \mathrm{l})\end{array}$ \\
\hline M1 & 93.28 & 609 & 0.638 & 20 \\
M2 & 93.28 & 1129 & 0.638 & 20 \\
M3 & 93.28 & 1654 & 0.638 & 20 \\
M4 & 93.28 & 2174 & 0.638 & 20 \\
M5 & 93.28 & 1654 & 2.41 & 20 \\
M6 & 93.28 & 1654 & 4.12 & 20 \\
S1 & 90.02 & 1654 & 3.979 & 20 \\
S2 & 149.6 & 1654 & 3.979 & 20 \\
S3 & 520.9 & 1654 & 3.979 & 20 \\
S4 & 1431.8 & 1654 & 3.979 & 20 \\
\hline
\end{tabular}

were calculated from the mass delivered at the manifold exit, over times greater than $1 \mathrm{~min}$. Each injection using each manifold was performed at least three times and the resulting peaks recorded at a chart speed of 6 or $30 \mathrm{~cm}$ $\min ^{-1}$. The carrier was then replaced with the tartrazine solution to obtain a steady state absorbance. Representative peaks were then scanned and converted to data points which were then saved in a Cricketgraph file. These data values were then scaled and normalised by obtaining appropriate scaling factors from the original chart recordings.

\section{Algorithm}

The development of the program involved the trial of a number of versions, the correction of which produced the current version from which the results in this paper were obtained.

On starting a run, the program requests the length and internal diameter, in millimetres, of manifold tubing used between valve and detector. Two values may be input for each. The sample volume and detector volume (in $\mu \mathrm{l}$ ), and the flow-rate (in $\mathrm{ml} \mathrm{min}^{-1}$ ) are then requested. The volume (in $\mu \mathrm{l}$ ) of each component (valve, manifold tube, detector) is then calculated and the flow-rate converted to $\mu \mathrm{s} \mathrm{s}^{-1}$. These volumes are then converted back to lengths by dividing by a normalised area of $\pi$. The manifold is then considered to be divided into segments longitudinally and radially the numbers of which are obtained using Eqn. 1.

$R=4.3976+4.0668 \log (L / F)$

where $R$ is the ratio of the number of radial segments, $S_{R}$, divided by the reciprocal of the normalised length of each longitudinal segment, $S_{L}, L$ is the total length of the normalised manifold and $F$ the velocity of the central stream line, assuming laminar flow. This equation was obtained empirically as described later in the results section. The number of segments through which the central stream line passes in one second, is the velocity of that stream line multiplied by $S_{L}$. An initial value of 1 is assigned to $S_{L}$ so that the time required to traverse each segment is $1 / F$ seconds. Then $S_{R}$ is calculated. Unless $S_{R}$ is within 0.25 of a whole number, the value of $S_{L}$ is decreased by 0.001 (effectively increasing segment length) and $S_{R}$ is recalculated. Once a satisfactory value for $S_{R}$ is obtained it is rounded to a whole number. For subsequent steps in the program to work, $S_{R}$ must not be less than 3 . If such a value is obtained, $S_{R}$ is set to 3 and $S_{L}$ is calculated.

The volumes of the resultant radial segments are then calculated using Eqn. 2.

$$
\begin{aligned}
V_{n+1}= & \pi\left\{\left[\left(S_{R}-n\right) / S_{R}\right]^{2}\right. \\
& \left.-\left\{\left[S_{R}-(n+1)\right] / S_{R}\right\}^{2}\right\}
\end{aligned}
$$

where $V_{n+1}$ is the volume of the segment and $n$ the integral distance from the wall such that $n+1$ defines the segment number. The outer radius of each segment is $\left(S_{R}-n\right) / S_{R}$ and $\left[S_{R}-\right.$

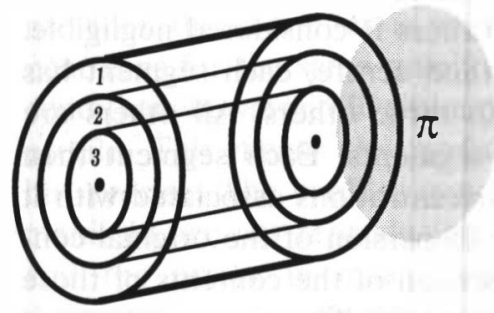

Fig. 2. Segmentation of the tube. Segment numbers increase from the wall. The tube is normalised to have a cross sectional area of $\pi$. 
$(n+1)] / S_{R}$ is the inner radius of each segment. This is summarised in Fig. 2.

When liquid flows in tubing of dimensions typically used for flow injection analysis, a parabolic flow profile is induced by laminar flow [22]. The distance $d$ travelled by material in each radial segment can thence be calculated as a proportion of the length of a segment from the parabolic profile defined by Eqn. 3 .

$d=\left[2(n+0.5) / S_{R}\right]-\left[(n+0.5) / S_{R}\right]^{2}$

In this equation, as in Eqn. 2, n+1 is the segment number, which has its centre a distance of $(n+0.5) / S_{R}$ from the tube wall.

The number of longitudinal segments for each section of the manifold is calculated by multiplying the normalised volumes by $S_{L}$ and rounding to a whole number. Therefore if the value of $S_{L}$ is 1 the number of segments equals one per normalised length unit. Two matrices are then generated both of which represent the manifold divided radially by $S_{R}$ and longitudinally by $S_{L}$.

The program then requires that a choice be made between whether reverse [23] or conventional flow-injection analysis (FIA) is modelled. In the conventional case, values of 1 multiplied by the segment volume are put in the starting array elements (representing the sample loop), and 0 in all other array elements. If reverse FIA is required, the values are reversed.

The iterative process of sample transport is then initiated. Diffusion is modelled first by considering the contents of a segment to disperse into those surrounding it. This leads to the concentration of one segment being averaged over the volume of itself and the volumes of those surrounding it (i.e., those with a common surface). Diffusion across corners is considered negligible. Thus at the walls and centre, each segment has common faces with three others. All others are bounded by four segments. Each segment then has four or five concentrations associated with it obtained from the dispersion of the original contents and the dispersion of the contents of those segments surrounding it. These concentrations are then summed for each segment and the values obtained are put in the second array. This ensures that the contents of the original array are not disturbed for the calculation of diffusion when another segment is considered. Once the diffusion process has been modelled for the whole manifold, convection is modelled by removing a portion of a segment according to the parabolic profile described by Eqn. 3. This removed portion is then added to the portion that remains in the neighbouring downstream segment, effectively moving some of the contents of a segment towards the detector. These new values replace the values in the original array. The concentration in the model detector is then calculated by summing the contents of that part of the array corresponding to the detector and dividing by the volume.

The model processes of diffusion, convection and detection are then repeated a number of times proportional to the length of the manifold in order that all the sample passes through the detector. As each transfer can represent a fraction of a second a large manifold or a highly segmented one would produce an unnecessary amount of data. Therefore, data values of time and absorbance are stored when the integer of time changes i.e., approximately every second. To ensure that the true peak maximum is not lost during this process, it is saved separately.

Two other routines included in the program but not implemented are transport of material by convective flow and calculation of product concentration when the injected and carried species react. (A listing of the program is available on request from the authors.)

The program was run and each time, the different dimensions of the real manifolds were used as input. The model was also used to predict the peaks obtained if flow cells with different volumes were used. The arrays of data were then imported into the corresponding Cricketgraph file of data obtained for real manifolds.

Manifolds described by Stone [17] were also modelled but peak heights only were recorded.

\section{RESULTS AND DISCUSSION}

One of the programs devised during development of the one described here operated in a 
similar manner to that described by Golay and Attwood [20,21]. In their model, transfer by convection could occur from one element to another over a distance of many elements. Diffusion was modelled by distributing fractions of the content of a segment to the adjacent segments. This produced double or humped peaks. These irregularities were considered to occur because of the discrete nature of the segments which caused the artificial separation of material as it is moved a number of segments away from its neighbours. Double peaks are predicted under certain diffusion-convection flow regimes [1,24], but are only seen for short, straight open tubular flow manifolds under a limited range of operating conditions $[1,25]$. The double peaks which are seen when using real, non-ideal, manifolds [19] may be due to the existence of more than one type of flow regime (due to "dead" volumes within the manifold), as proposed by Stone [14] and Stone and Tyson [16].

When the program was under development, it was noted that the peak height decreased as the ratio of radial to linear segments was increased. As peak height changes with flow-rate as well as with manifold dimensions $[14,16]$, this ratio was changed until good agreement was obtained for peak heights obtained using real manifolds with different flow-rates and manifold lengths. The ratios obtained were plotted against the log to the base 10 of total manifold length $L_{\mathrm{w}}$ divided by flow-rate $F$. This plot is shown in Fig. 3, and the line fitted by least squares produced Eqn. 1 with a correlation coefficient of 0.988 . As the residence time increases, longitudinal diffusion may become significant compared to radial diffusion. As $R$ increases, the number of radial segments increases compared to the number of linear segments causing the averaging of the concentrations to be performed over a larger longitudinal distance reflecting the increase in importance of longitudinal diffusion.

If a different valve or detector geometry were used, as was the case for the experiments described in Ref. 14, any localised mixing may be different and Eqn. 1 may not be valid. However a similar equation would be expected.

After initial conditions had been declared, the

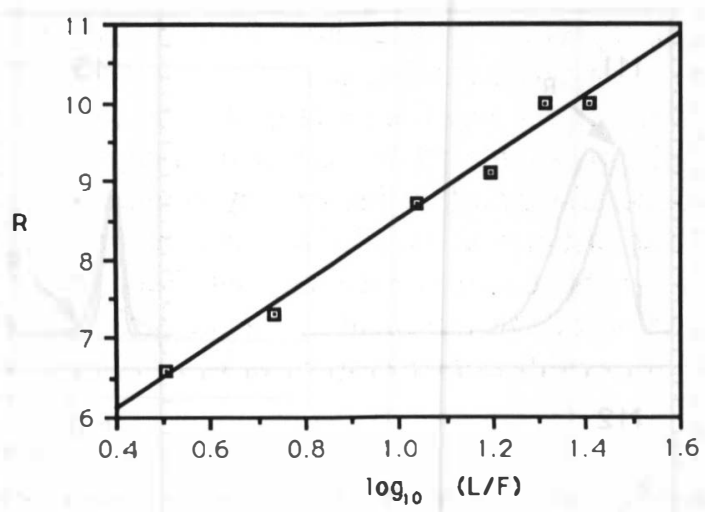

Fig. 3. A plot of the ratio of the number of radial segments to longitudinal segments, $R$, vs. the log of total manifold length, $L$, divided by the velocity of the central stream line, $F$.

peak profile data was obtained after a time that was proportional to the residence time of the sample in the manifold and varied from less than $1 \mathrm{~min}$ to about $30 \mathrm{~min}$. This time was also affected by the number of users using the computer at any one time. Compared to Monte Carlo type simulations [8], which can take several hours, the computing power and time that the program requires to produce satisfactory data is small.

Peak profiles both for real manifold generated data and for the corresponding models were plotted using Cricketgraph and are presented in Fig. 4. When comparing the plots of peak profiles, the sources of errors in each profile must be taken into account. As the modelling routine requires whole numbers of segments for each section of the manifold, some errors in volumes used will occur, and cause slight distortion in the peak shape, height and arrival times. It can be expected that if the program is run with the same input data, the output data will be the same provided the computer is working properly. Repeat runs for the same manifold under the same conditions however will produce exactly the same profile. This means the modelling is inaccurate but precise. The profiles generated using a real manifold are not reproducible for each injection and the errors in measuring volume injected, detection volume, flow-rate, tubing inconsistencies and the occurrence of localised mixing will produce inaccuracies. The profiles produced 


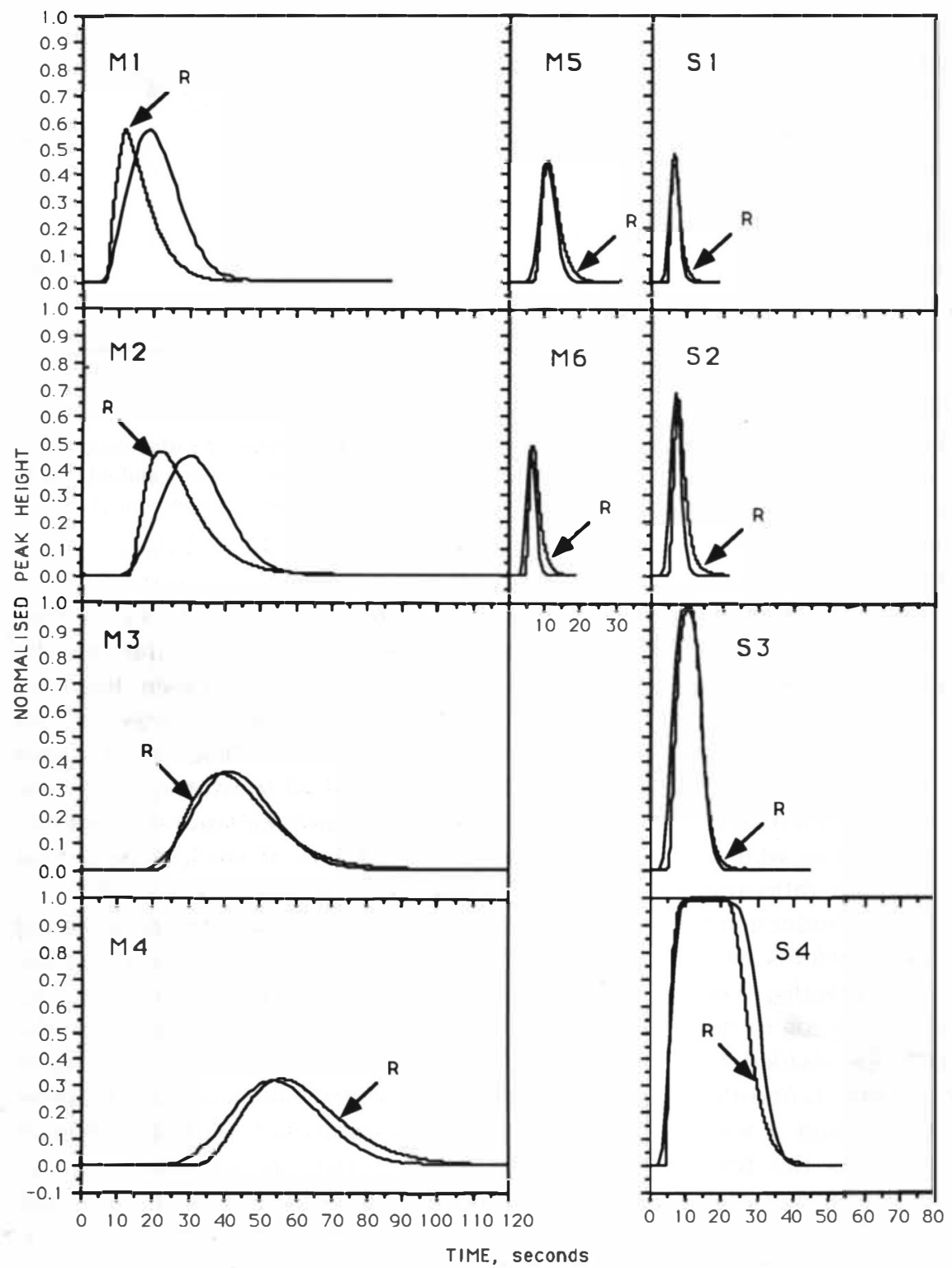

Fig 4. The peaks produced using real manifolds (labelled R) and the model. See Table 1 for a description of manifolds M1 to S4.

therefore will be both inaccurate and imprecise. However, the peak profiles, Fig. 4, can be compared for the traits of peak height, appearance time of the start of the peak and peak maximum and peak shape.

For the various sample volumes injected, peak heights are predicted accurately but there appears to be a slight error in the arrival and appearance times. One possible reason for this is the relative weight given by the model to the axial diffusion process. If this is too high, then the predicted appearance times will be shorter than the experimental times, which is what is observed. Peak shapes are in reasonably good agreement.

For varying tube length, arrival time and peak height are in good agreement but the peak shape 

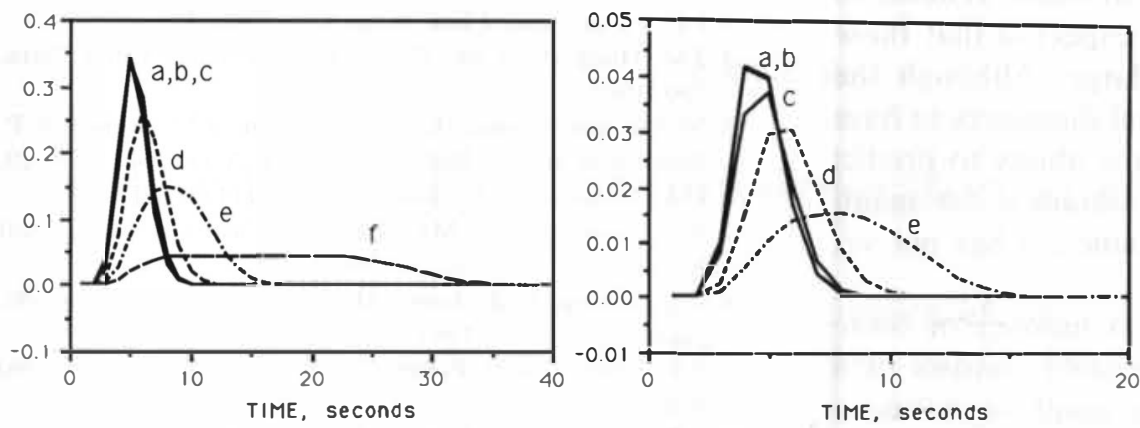

Fig. 5. The peaks produced by modelling different detector volumes for a manifold consisting of $1000 \mathrm{~mm}$ of $0.5 \mathrm{~mm}$ i.d. tubing: (i) Injected volume $50 \mu \mathrm{l}$. (ii) Injected volume $10 \mu \mathrm{l}$. Detector volumes (a) 3, (b) 8, (c) 20, (d) 100, (e) 300 and (f) $1000 \mu \mathrm{l}$.

and hence the appearance time is not so well predicted. This is improved however, at higher flow-rates.

Increasing the flow-rate sharpens the peaks. This trend is reflected well by the model, but there is a tendency for the model to shorten the arrival and appearance times.

The trends observed for increasing detector volume, shown in Fig. 5, are those expected, in that the peaks become lower and broader. It should be noted that even using the crude plots of the modelled data in Fig. 5, no significani change in peak shape or height is apparent unless the detector volume exceeds the injected volume. Unfortunately, a range of flow cells is not available so a comparison of the model data with profiles obtained using real manifolds cannot, at present, be made.
The manifolds used by Stone [14] are summarised in Table 2 along with the resultant normalised peak heights and those obtained using this model. The flow cell in the detector used by Stone was of a very small volume and could not be modelled. However, as stated previously, the model does not distort the peaks if $V_{\mathrm{d}}<V_{\mathrm{i}}$ and therefore a volume of $10 \mu \mathrm{l}$ was used. The model peak heights are close to those obtained by Stone, but little precision data is available to be able to see how significant the differences are between the model and the manifold data. Although a different valve was used in the manifold used by Stone, Eqn. 1 appears valid for his system.

\section{Conclusions}

The model developed is able to predict peak height for the systems used. With minor modifi-

TABLE 2

Manifolds modelled from Ref. 14. (Tubing internal diameter was $0.58 \mathrm{~mm}$ throughout)

\begin{tabular}{cllllll}
\hline $\begin{array}{l}V_{\mathrm{s}}{ }^{\mathrm{a}} \\
(\mathrm{ml})\end{array}$ & $\begin{array}{l}L_{\mathrm{m}}{ }^{\mathrm{a}} \\
(\mathrm{mm})\end{array}$ & $\begin{array}{l}F^{\mathrm{a}} \\
\left(\mathrm{ml} \mathrm{min}{ }^{-1}\right)\end{array}$ & $\begin{array}{l}V_{\mathrm{d}}{ }^{\mathrm{a}} \\
(\mathrm{ml})\end{array}$ & $\begin{array}{l}V_{\mathrm{d}}, \text { modelled } \\
(\mathrm{ml})\end{array}$ & $\begin{array}{l}\text { Peak } \\
\text { height }{ }^{\text {a }}\end{array}$ & $\begin{array}{l}\text { Peak height, } \\
\text { modelled }\end{array}$ \\
\hline 67.3 & 1000 & 2.11 & $<1$ & 10 & 0.31 & 0.36 \\
67.3 & 1700 & 2.11 & $<1$ & 10 & 0.25 & 0.26 \\
67.3 & 3500 & 2.11 & $<1$ & 10 & 0.18 & 0.17 \\
113 & 3350 & 1.00 & $<1$ & 10 & 0.31 & 0.26 \\
113 & 1850 & 1.00 & $<1$ & 10 & 0.36 & 0.37 \\
67.3 & 3500 & 2.22 & $<1$ & 10 & 0.18 & 0.17 \\
67.3 & 1000 & 2.22 & $<1$ & 10 & 0.31 & 0.40 \\
113 & 3350 & 5.00 & $<1$ & 10 & 0.36 & 0.34 \\
113 & 1000 & 5.00 & $<1$ & 10 & 0.61 & 0.62 \\
\hline
\end{tabular}

\footnotetext{
${ }^{a}$ From Ref. 14.
} 
cations, it may be extended to other systems of valves or detectors. It is not expected that these changes would need to be large. Although the model normalises tube internal diameters to have a cross sectional area of $\pi$, the ability to predict accurately the peak heights obtained for manifolds of different internal diameters has not yet been investigated.

As the model consists of a number of finite segments whereas a real manifold consists of a infinite number of infinitely small segments, a compromise in the prediction capabilities of the model must be expected. In this case the model was optimised in order that peak height was predicted reliably, but another parameter could have been chosen such as peak widths or shape. The trend shown by the model for various detector volumes, indicates that in order to obtain reliable and known values of dispersion due to mixing of carrier and injectate rather than detector averaging effects, the injected volume should exceed the detector volume. In most practical FIA systems, this is normally the case and indicates many of the dispersion values quoted are due to dispersion outside the detector.

It is hoped that further work with other manifolds and components will yield a model that is applicable to most systems. This model could be extended to timed injection, manifolds with mixing chambers, multi-line manifolds, chemical reaction and varying concentrations of injected species.

\section{REFERENCES}

1 J.T. Vanderslice, K.K. Stewart, A.G. Rosenfeld and D. Higgs, Talanta, 28 (1981) 11.
2 J.F. Tyson and A.B. Idris, Analyst, 106 (1981) 1125.

3 J.F. Tyson, Anal. Chim. Acta, 179 (1986) 131.

4 J.M. Hungerford and G.D. Christian, Anal. Chim. Acta, 200 (1987) 1.

5 M.A.J. van Opstal, J.J.F. van Veen, J.M. Reijn, W.P. Bennekom and A. Bult, Anal. Chim. Acta, 204 (1988) 29.

6 D.C. Stone and J.F. Tyson, Analyst, 114 (1989) 1453.

7 M.A. Gomez-Nieto, M.D. Luque de Castro, A. Martin and M. Valcarcel, Talanta, 32 (1985) 319.

8 C.D. Crowe, H.W. Levin, D. Betteridge and A.P. Wade, Anal. Chim. Acta, 194 (1987) 49.

9 S.D. Kolev and E. Pungor, Anal. Chim. Acta, 185 (1986) 315.

10 S.D. Kolev and E. Pungor, Anal. Chem., 60 (1988) 1700.

11 S.D. Kolev and E. Pungor, Anal. Chim. Acta, 208 (1988) 117.

12 S.D. Kolev and E. Pungor, Anal. Chim. Acta, 208 (1988) 133.

13 I.C. van Nugteren-Osinga, M. Bos and W.E. van der Linden, Anal. Chim. Acta, 214 (1988) 77.

14 D.C. Stone, Models for Dispersion in Flow Injection Analysis, a Doctoral Thesis, Loughborough University of Technology, 1987.

15 D.C. Stone and J.F. Tyson, Anal. Chim. Acta, 179 (1986) 427.

16 D.C. Stone and J.F. Tyson, Analyst, 112 (1987) 515.

17 S.R. Bysouth, Standard and Sample Manipulation for Calibration in Flame Atomic Absorption Spectrometry, a Doctoral Thesis, Loughborough University of Technology, 1988.

18 J.F. Tyson, Analyst, 115 (1990) 587.

19 E. Beinrohr, P. Csemi and J.F. Tyson, J. Anal. At. Spectrom., 6 (1991) 307.

20 M.J.E. Golay and J.G. Atwood, J. Chrom., 186 (1979) 353.

21 J.G. Atwood and M.J.E. Golay, J. Chrom., 218 (1981) 97.

22 W.L. McCabe, J.C. Smith and P. Harriot, Unit Operations of Chemical Engineering, Fourth Edition, McGraw Hill, Singapore, 1985.

23 J.F. Tyson and A.B. Idris, Analyst, 106 (1981) 1125.

24 W.N. Gill and V. Ananthakrishnan, A.I.Ch.E.J., 13 (1967) 801.

25 J.M. Reijn, W.E. van der Linden and H. Poppe, Anal. Chim. Acta, 126 (1981) 1. 